\title{
A PROSPECTIVE OBSERVATIONAL STUDY ON DRUG UTILIZATION PATTERN IN PATIENTS WITH CHRONIC KIDNEY DISEASE AT A TERTIARY CARE HOSPITAL
}

\section{Sujana C N}

\section{Dr. Niranjan M R*}

Department Of Pharmacology, Mysore Medical College And Research Institute, Mysore, Karnataka, India.

Department Of Nephrology, Mysore Medical College And Research Institute, Mysore, Karnataka, India. ${ }^{*}$ Corresponding Author

\section{Parashivamurthy B M}

Department Of Pharmacology, Mysore Medical College And Research Institute, Mysore, Karnataka, India.

ABSTRACT Background: Poly-pharmacy being most common in chronic kidney disease patients (CKD). The present study was carried out to analyse current prescribing trends in the management of CKD patients and to compare it with WHO Core Indicators. Methods: A prospective observational study was carried out for three months (15th July 2019 - 15th October 2019) after Institutional Ethics Committee approval at a tertiary care hospital. Patients diagnosed with CKD by treating Nephrologist were included and their prescriptions (OPD card) were analysed to study the prescribing patterns. Results: A total of 60 cases were analysed during the study, of which $73.3 \%$ were males and $26.7 \%$ were females. The common comorbidities were hypertension $(36.6 \%)$, diabetes $(36.6 \%)$, other cardiovascular diseases $(26.6 \%)$, anaemia $(3.33 \%)$, Ca Cervix (1.67\%), osteoarthritis (6.67\%). Among drugs Antihypertensive drugs (40.9\%) were the most commonly used drugs, followed by, Anti-diabetic drugs, calcium salts and multi-vitamins (19.7\%), oral iron supplements and erythropoietin (13.4\%) and ulcer protective (6.1\%). Conclusions: Polypharmacy being followed in these CKD patients were necessary for multiple conditions of patient, supplemental drugs decreased adverse effects on initial drug and they yielded synergistic effects. Maximum numbers of drugs were prescribed from anti-diabetic, antihypertensive, supplemental drugs and other cardiovascular class of drugs. The principle of rational prescribing was followed. The right choice of drugs and in appropriate doses will reduce the incidence of nephrotoxicity and ultimately result better clinical outcomes.

\section{KEYWORDS :Chronic kidney disease (CKD), Drug prescribing pattern, polypharmacy.}

\section{INTRODUCTION}

Chronic kidney disease (CKD) is a worldwide threat to public health and has a risk-multiplier effect on major noncommunicable diseases, including cardiovascular diseases.

The Global burden of diseases (GBD) 2015 study also estimated that, in 2015, 1.2 million people died from kidney failure, an increase of $32 \%$ since 2005. In 2010, an estimated 2.3-7.1 million people with end-stage kidney disease died without access to chronic dialysis. Additionally, each year, around 1.7 million people are thought to die from acute kidney injury. Overall, therefore, an estimated 5-10 million people die annually from kidney disease. ${ }^{1}$ Accordingly the Kidney disease must be managed properly by proper treatment and preventive measures should be employed, so that patient doesn't develop end stage renal disease (ESRD).

The decline in glomerular filtration rate (GFR) and increase in tubular secretion of endogenous substances (and medications) increase risk for adverse drug-related kidney effects. GFR reduction and increased drug exposure in a reduced number of functioning nephrons and ischemia preconditioned tubular cells, and more robust oxidative injury response to various medications by the kidney may enhance kidney tubular toxicity. ${ }^{2}$

Polypharmacy may lead to adverse reactions if they are left unmonitored. Choosing of drug to treat already damaged renal morphology play a vital role. Guidelines should be followed to choose renal safe drugs in CKD patients, dose adjustment to be done, risk-benefit must be analysed before prescribing drugs to the patient.

CKD has multifactorial causation and the complications like anaemia, bone and mineral disease are more often associated. Hypertension and diabetes, recurrent infections along with an inappropriate prescription of drugs are the leading factors resulting in the increasing incidence of $\mathrm{CKD} .^{3}$
World Health Organization (WHO) has defined Drug utilization study (DUS) as "The marketing, distribution, prescription and uses of drugs in a society with special emphasis on the resulting medical, social and economic consequences." The principle aim of the drug utilization research is to facilitate the rational use of the drugs. It is very difficult to improve prescribing habits, without knowing about the pattern of drug use in the patients. ${ }^{4}$

In the absence of a renal registry, the exact disease burden of CKD/ESKD in the Indian population cannot be assessed accurately. Hence this study is undertaken to analyse current prescribing trends. A study of the prescription patterns of drugs would be of interest to prevent drug related adverse outcomes.

\section{METHODS}

The present study is Prospective observational study conducted over a period of 3 months from $15^{\text {th }}$ July 2019 to $15^{\text {th }}$ October 2019.

The estimated sample size was 60 based on the 12 month prevalence $(p)$ of CKD $-17.2 \%$.

After obtaining clearance from the Institutional Ethics Committee, subjects attending nephrology OPD department at Krishna Rajendra Hospital, attached to Mysore Medical College and Research Institute, Mysore, diagnosed with CKD by the treating Nephrologist, were included in the study after obtaining a written informed consent. The socio-demographic data along with other relevant details of the study subjects was collected from patient's OPD card and their prescriptions were analysed as per "WHO drug use indicators included"

Eligibility/ (Inclusion Criteria)

1. Patients of all sex,

2. Age 18 years or more,

3. Diagnosed by the clinician to have chronic kidney disease 
and currently on drug treatment will be included in study.

Exclusion Criteria

1. Pregnant and lactating women

2. Surgical conditions like kidney stone, tumors and trauma will be excluded.

\section{RESULTS}

After analysing 60 cases during the study the results are as follows

\section{Demographic profile:}

Out of 60 cases male predominance of $73.3 \%$ was seen and females were $26.7 \%$. The mean age was 52 years ( $23 \mathrm{yr}-75 \mathrm{yr}$ ). Most of the cases fell under 30 to 60 years of age i.e. 36(n) $60 \%$, more than 60 years of age were 20 (n) $33.33 \%$, less than 30 years of age were $4(n) 6.67 \%$.

\section{Disease pattern:}

The common comorbidities were hypertension (36.6\%), diabetes $(36.6 \%)$, other cardiovascular diseases $(26.6 \%)$, ancemia (3.33\%), Ca Cervix (1.67\%), osteoarthritis (6.67\%) and others. Other comorbidities include dyslipidaemia, hypothyroidism, infectious conditions (URTI, UTI and others.), and pancreatitis.

\section{WHO core indicators:}

Analysis of WHO core drug prescribing indicators showed that

1. The average number of drugs prescribed per patient was 6.43,

2. Percentage of drugs prescribed by generic name was $18.51 \%$,

3. Percentage of patients with an injection prescribed was $6.6 \%$,

4. Percentage of drugs prescribed from essential medicine list was $72.8 \%$,

5. Percentage of drugs prescribed as fixed dose combinations was $7.9 \%$ and

6. Percentage of patients prescribed with an antibiotic was $12.8 \%$.

\section{Drug utilization pattern:}

The total number of drugs encountered was 386. Out of which Antihypertensive drugs (40.9\%) were the most commonly used drugs, followed by anti-diabetic drugs (38.7\%), calcium salts and multi-vitamins (19.7\%), oral iron supplements and erythropoietin (13.4\%), ulcer protective $(6.1 \%)$ and others. According to Anatomic Therapeutic Classification of drugs by WHO, it showed ATC class code - A and C were most commonly used followed by ATC class code-B and R. (Table 1 and Table 2)

Table 1: Drug classification according to ATC classification.

\begin{tabular}{|l|l|l|l|}
\hline $\begin{array}{l}\text { ATC } \\
\text { Class } \\
\text { code }\end{array}$ & ATC Class & $\begin{array}{l}\text { No.of drugs } \\
(\mathrm{n}=386)\end{array}$ & $\begin{array}{l}\text { Percentage } \\
(\%)\end{array}$ \\
\hline A & $\begin{array}{l}\text { Alimentary tract and } \\
\text { metabolism }\end{array}$ & 169 & 43.78 \\
\hline B & $\begin{array}{l}\text { Blood and blood forming } \\
\text { products }\end{array}$ & 34 & 8.80 \\
\hline C & CVS & 108 & 27.98 \\
\hline D & Dermatology system & 0 & 0 \\
\hline G & $\begin{array}{l}\text { Genitourinary system and } \\
\text { sex hormones }\end{array}$ & 0 & 0 \\
\hline H & $\begin{array}{l}\text { Systemic hormonal } \\
\text { preparation }\end{array}$ & 0 & 0 \\
\hline J & $\begin{array}{l}\text { Anti- infectives for systemic } \\
\text { use }\end{array}$ & 9 & 2.33 \\
\hline L & $\begin{array}{l}\text { Anti-neoplastic and } \\
\text { immunomodulatory agent }\end{array}$ & 1 & 0.26 \\
\hline
\end{tabular}

\begin{tabular}{|l|l|l|l|}
\hline $\mathrm{M}$ & Musculoskeletal system & 44 & 11.34 \\
\hline $\mathrm{N}$ & Nervous system & 0 & 0 \\
\hline $\mathrm{P}$ & $\begin{array}{l}\text { Anti-parasitic products, } \\
\text { insecticides and repellents }\end{array}$ & 0 & 0 \\
\hline $\mathrm{R}$ & Respiratory system & 17 & 4.40 \\
\hline $\mathrm{S}$ & Sensory organ & 0 & 0 \\
\hline $\mathrm{v}$ & Various & 4 & 1.03 \\
\hline
\end{tabular}

Table 2: Therapeutic class wise drugs used

\begin{tabular}{|l|l|}
\hline ATC & ATC Subclass and drugs \\
\hline
\end{tabular}

\begin{tabular}{ll|l}
\hline A02 & Drugs for acid related disorders \\
\hline
\end{tabular}

A04 Antiemetics and antinauseants

Al0 Drugs used in diabetes

A $10 \AA$ - Insulins and analogues

A10B - Oral blood glucose lowering drugs

AlOX - Other drugs used in diabetes

\begin{tabular}{|l|l}
\hline All & Al lCC -Vitamin D and analogues \\
\hline
\end{tabular}

Al2 Mineral Supplements

Al2A - Calcium

Al2B -Potassium

B $\quad$ B01AC04 clopidogrel

B01AB05 enoxaparin

B03A - Iron preparations

B03B - Vitamin B12 and Folic ACID

B03XA01 -Erythropoietin

C COlDA08 antianginal

C02ACOl clonidine

C02DB02 hydralazine

C07AB02 metoprolol

C08CA5l amlodipine

C09DX07 irbesartan, amlodipine and

hydrochlorothiazide

C10BA08 atorvastatin

C08CA14 Cilnidipine

C09AA02 enalapril

C08CA04 nicardipine

C02CA01 prozosin

D D07AA01 prednisolone

\begin{tabular}{|l|l}
\hline M & M04AA03 febuxostat
\end{tabular}

M02AX06 tolperisone

\begin{tabular}{l|l}
$\mathrm{N}$ & $\mathrm{N} 02 \mathrm{AJ} 13$ tramadol and paracetamol
\end{tabular}

N06CA03 fluoxetine

\begin{tabular}{l|l}
\hline$R$ & R03AL10 formoterol and tiotropium bromide
\end{tabular} R02AB Antibiotics

V V04CX02 folic acid

\section{Other findings:}

1. No. of anti-hypertensive drugs per prescription -2.15

2. Patients prescribed with diuretics $-70 \%$

3. Number of drugs per patient (Table 3)

Table 3: Number of drugs per patient

\begin{tabular}{|l|l|}
\hline No. of Drugs Prescribed No of patients (n) & Percentage \% \\
\hline
\end{tabular}

\begin{tabular}{|l|l|l|}
\hline$\leq 5$ & 22 & 36.67 \\
\hline $6-10$ & 30 & 50 \\
\hline $11-15$ & 8 & 13.33 \\
\hline
\end{tabular}

\section{DISCUSSION}

According to the world health organization (WHO), CKD contributes to nearly 850,000 deaths per year worldwide. ${ }^{6}$ Drug utilization in CKD changes with time period, physician, disease conditions and population, which makes it important to study the drug utilization continuously over a period of time.? CKD patients must take medicines for lifelong, hence on a regular basis prescribing trends should be studied. ${ }^{8}$

CKD patients present with several co-morbidities such as hypertension, diabetes mellitus, coronary artery disease and infection. On the other hand, inappropriate use of drugs and poly-pharmacy makes CKD patients more vulnerable to complications and drug induced kidney diseases. ${ }^{9}$ 
Diabetes and hypertension have been reported to be the most common risk factors for $\mathrm{CKD}$, which are proven in the current study also. Thus multifactorial causation factors for developing chronic kidney disease are strongly agreed.

The cardiovascular conditions associated in the current study are apart from hypertension, ischemic heart disease, bradycardia (metopolol induced), coronary artery disease, angina atherosclerosis. The drugs used in CVS conditions were diuretics, beta blocker. Alpha blockers, ACE inhibitors, ARB inhibitors, calcium channel blockers, vasodilators, anticoagulants, statins, antianginal drugs.

To delay bone and mineral diseases patients were put mainly on calcium, phosphate binders, vitamin D.

The association of CKD with gender difference of male predominance in the present study is similar with Tamilselvan study. ${ }^{10}$

The average number of drugs per patient is about 6.12 and is similar to Tamilselvan study which stated $5.26 \pm 3.79$ drugs per patient. ${ }^{10}$

Tamilselvan study showed Anaemia and dyslipidaemia were fairly prevalent in the study population of CRF patients other than hypertension and diabetes mellitus. These findings are similar in the current study too. ${ }^{10}$

Erythropoietin (EPO) is normally produced by interstitial fibroblasts in the renal cortex, in close proximity to tubular epithelial cells and peritubular capillaries. ${ }^{10}$ In CKD patient because of loss of structural integrity of kidney EPO is lacking hence the patients develop anaemia and are treated mainly with injection Erythropoietin, followed by other treatments such as oral or parenteral iron supplementation, folic acid and blood transfusion. The present study also shows anaemia in $3.33 \%$ and the patients are treated accordingly as mentioned above.

The pattern of HTN observed in the present study is that most of patients have resistant HTN, hence they are put on multiple antihypertensive drugs. Average number of antihypertensive in the present study is about 2.15 per patient.

The antihypertensive drugs used in the present study are:

1. Calcium channel blocker- Cilnidipine, amlodipine, nicardipine

2. Loop diuretics-Torasemide, Furosemide

3. Thiazide- metolazone

4. $\mathrm{K}^{+}$sparing diuretic-spironolactone

5. Sympathetic inhibitors - metoprolol, prazosin, clonidine

6. ACE inhibitor-enalapril

7. Vasodilator-arterial-Hydralazine

To delay the progression of kidney disease the patients were put on N-acetylcysteine and Taurine drugs which showed promising results in most of the patients. Even Probiotics showed promising results when used in CKD patients in the current study.

Chronic kidney disease is a problem of epidemic proportion in India and with increasing diabetes burden, hypertension and growing elderly population it is further going to increase. Managing patient population of CKD should be aimed at proper utilization of available drugs and preventing progression of disease to ESRD.

The aims of CKD management in Pharmacology are to: ${ }^{11}$

a. Prevent/delay disease progression

b. Prevent/minimise the cardiovascular complications of kidney disease c. Treat anaemia

d. Treat mineral and bone disorders

e. Treat the underlying kidney disease if possible

f. In order to prevent both CKD progression and cardiovascular events, it is important to control both hypertension and diabetes.

Present study illustrates the current day scenario of CKD patients attending Nephrology OPD in a tertiary care hospital, prescribing trends of the physicians in managing these patients with co-morbidities and disease related complications. Present study provides the baseline data and would help build data for carrying out further drug utilization studies.

\section{ACKNOWLEDGEMENTS}

We acknowledge Head of Department of Pharmacology, Head of Department of Nephrology for granted permission and valuable support.

\section{DECLARATIONS}

Funding: None

Conflict of interest: None

Ethical approval: Approved from Institutional ethical committee.

\section{REFERENCES}

1. Valerie A, Marcello, T and John W S. The global burden of kidney disease and the sustainable development goals. Bulletin of the World Health Organization 2018; 96: 414-422D. doi: http://dx.doi.org/10.2471/BLT.17.206441. Accessed on 10 July 2019

2. Perazella MA: Drug-induced renal failure: Update on new medications and unique mechanisms of nephrotoxicity. AmJMedSci 325: 349-362, 2003.

3. Kappel J, Calissi P. Nephrology: 3. Safe drug prescribing for patients with renal insufficiency. CMAJ 2002; 166:473-7.

4. W H D D U G U T I L I Z A T ION RE S E A R C H 2013 https://apps.who.int/medicinedocs/en/d/Js4876e/2.html Accessed on 30th May 2019.

5. Singh AK, Farag YMK, Mittal BV. Epidemiology and risk factors of chronic kidney disease in India - results from the SEEK (Screening and Early Evaluation of Kidney Disease) study. BMC Nephrology 2013, 14:114.

6. World Health Organization: Burden of Disease Project. Available from: http://www3.who.int/whosis/menu.cfm

7. Laporte JR, Orme ML. Drug utilization and the teaching of rational drug use. WHO Reg Publ Eur; 1993; Ser 45: 183-91.

8. Ahlawat R, D'cruz S and Tiwari P. Drug Utilization Pattern in Chronic Kidney Disease Patients at a Tertiary Care Public Teaching Hospital: Evidence from a Cross- Sectional Study . Journal of Pharmaceutical Care \& Health Systems. 2016; volume3, issue 1;2376-0419.

9. Manley H J, Debra K, Drayer D K, Richard S, Muther R S. Medication related problem type and appearance rate in ambulatory hemodialysis patients. BMC Nephrology 2003; 4:10

10. Tamilselvan T, Veerapandiyan A K, Karthik N. Study on drug utilization pattern of chronic renal failure patients in a tertiary care hospital. Int J Pharm Pharm Sci, vol 6, issue 9, 482-484

11. Renal Medicine. David Oliveira, Debasish Banerjee, Joyce Popoola, Iain A.M. MacPhee, Seema Shrivastava, et al. Chapter 4 pg 76 